\section{Schwangerschaftstest nach Aschheim und Zondek}

T. Arndt

Bioscientia Institut für Medizinische Diagnostik GmbH, Ingelheim, Deutschland

Synonym(e) Aschheim-Zondek-Schwangerschaftstest

Englischer Begriff Aschheim-Zondek pregnancy reaction; Aschheim-Zondek reaction

Definition Heute obsoleter biologischer Test zum Nachweis einer Schwangerschaft unter Einsatz von Mäusen.

Beschreibung Nach den Gynäkologen Selmar Aschheim (1878-1965) und Bernhard Zondek (1891-1966) benanntes, früher häufig verwendetes Verfahren zur Schwangerschaftsfrüherkennung. Infantilen weiblichen Mäusen wird subkutan Schwangerenharn injiziert. Wachstum der Ovarien und Ovulation nach 4-5 Tagen zeigten eine Schwangerschaft an. Die Genauigkeit der Vorhersage lag bei $98 \%$. Im Vergleich zum Bitterlingstest ( $\triangleright$ Bitterlingstest zum Nachweis einer Schwangerschaft) war der Maustest also zuverlässiger. Die längere Ansprechzeit und die Notwendigkeit der Tötung der Versuchstiere waren Nachteile der Methode.

Varianten des Tests unter Verwendung anderer Tierspezies wie Krallenfrosch (6 Stunden Ansprechzeit), männlichen Fröschen (Auftreten von spermahaltigem Urin 2-4 Stunden nach Harninjektion) und der Deutschen Kröte (Bufo vulgaris, zur Ablösung von Krallenfroschimporten) mit Vermeidung der Obduktion waren Entwicklungsstufen auf dem Weg zum heutigen Schwangerschaftstest mit der spezifischen Bestimmung von Choriongonadotropin (HCG; > Choriongonadotropin, humanes) im Schwangerenharn.

Anmerkung: Vor 3000 Jahren sollen die Ägypter am beschleunigten Keimen/Wachstum von mit Schwangerenharn begossenem Getreide (Weizen, Gerste) mit $75 \%$ Treffsicherheit eine Schwangerschaftsfrühdiagnose erreicht haben.

\section{Literatur}

Aschheim S, Zondek B (1928) Die Schwangerschaftsdiagnose aus dem Harn durch Nachweis des Hypophysenvorderlappenhormons. Klin Wochenschrift 7(1404-1411):1453-1457

Schlögl HA (2006) Das alte Ägypten. Geschichte und Kultur von der Frühzeit bis zu Kleopatra. C. H. Beck, München, S 167 